

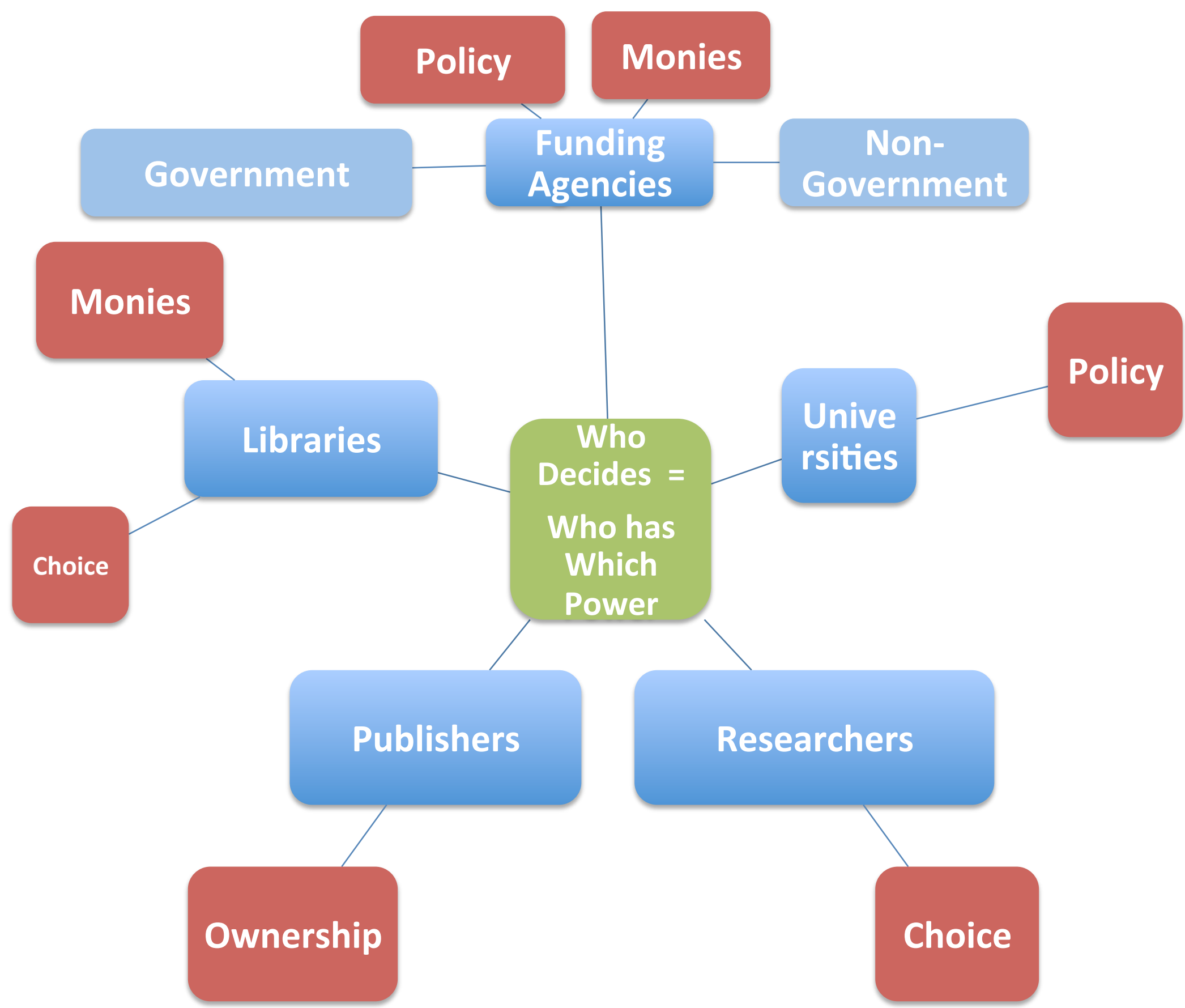




\section{Multiple Stakeholders Require}

a

\section{Convening Power}

- Professional Associations: Libraries, Universities, Societies

- Funders, Standards Bodies, Inter-Governmental Organizations... Independent organizations with stature, reputation, and a will to action 


\section{Key Goals}

- For the journal literature:

- An economically feasible, sustainable move to Open Access, preserving quality and ensuring preservation and access

- For scholarship generally:

- To take actions that will improve the creation and dissemination of new knowledge 


\section{Proposal: Models Where Powers are Combined}

\section{Research Evaluation}

\section{Alternative Models}

3. Global "Flip" 


\section{Evaluation}

Universities \& Research Communities

Convened by

Professional Associations

Policy, Choice

Fairly

Considering OA in the Evaluation

Process
Alternative / Article-Level

Metrics
Rewarding

(More)

Openness
Leveraging

Repositories 


\section{Alternative Models}

(Society) Publishers, Research Communities, Libraries

Convened by

Any of the above, or via independent grassroots action

Funding, Ownership, Choice

Incubate new avenues to disseminate scholarly work Lower cost OA -- Newer platforms

e.g., Open Library Humanities, LingOA, Libraria 


\section{Global "Flip"}

\section{Flip = Conversion of Existing Journals to Open Access}

\section{Libraries, Publishers, and Funders \\ Convened by \\ Organizations with global standing}

\section{Money, Choice, and Ownership}

Redirect Subscription Funding Toward Open Access Publication
Predictable and Sustainable Costs, Budgets, and Income 


\section{Example: Research Evaluation}

National Professional Association

- Convene: Learned Societies and Researchers, Others

\section{Charge a task force to}

- Conduct an environmental scan of alternative metrics initiatives and propose a roadmap for further action 


\section{Example: Global Flip}

- Convenes a meeting of interested stakeholders to formulate an initiative

Stakeholders

pledge interest in

moving forward

Analyze subscription spend and

publishing output by institution / country
- Analytical capability offered by MPDL, UC Pay It Forward project tools
Offset deals entered into
- With development of migration paths and best practices, while avoiding restraint of trade 


\section{Who Decides?}

- Ivy Anderson, California Digital Library

- Mel DeSart, Univ Washington

- Lee Cheng Ean, National Univ of Singapore

- Remi Gaillard, Univ Pierre and Marie Curie

- Susan Gibbons, Yale - Adam Huftalen, RELX

- Joan Lippincott, CNI

- Salvatore Mele, CERN

- Ralf Schimmer, Max Planck Digital Library

- Deborah Stine, Carnegie Mellon

- John Vaughn, AAU

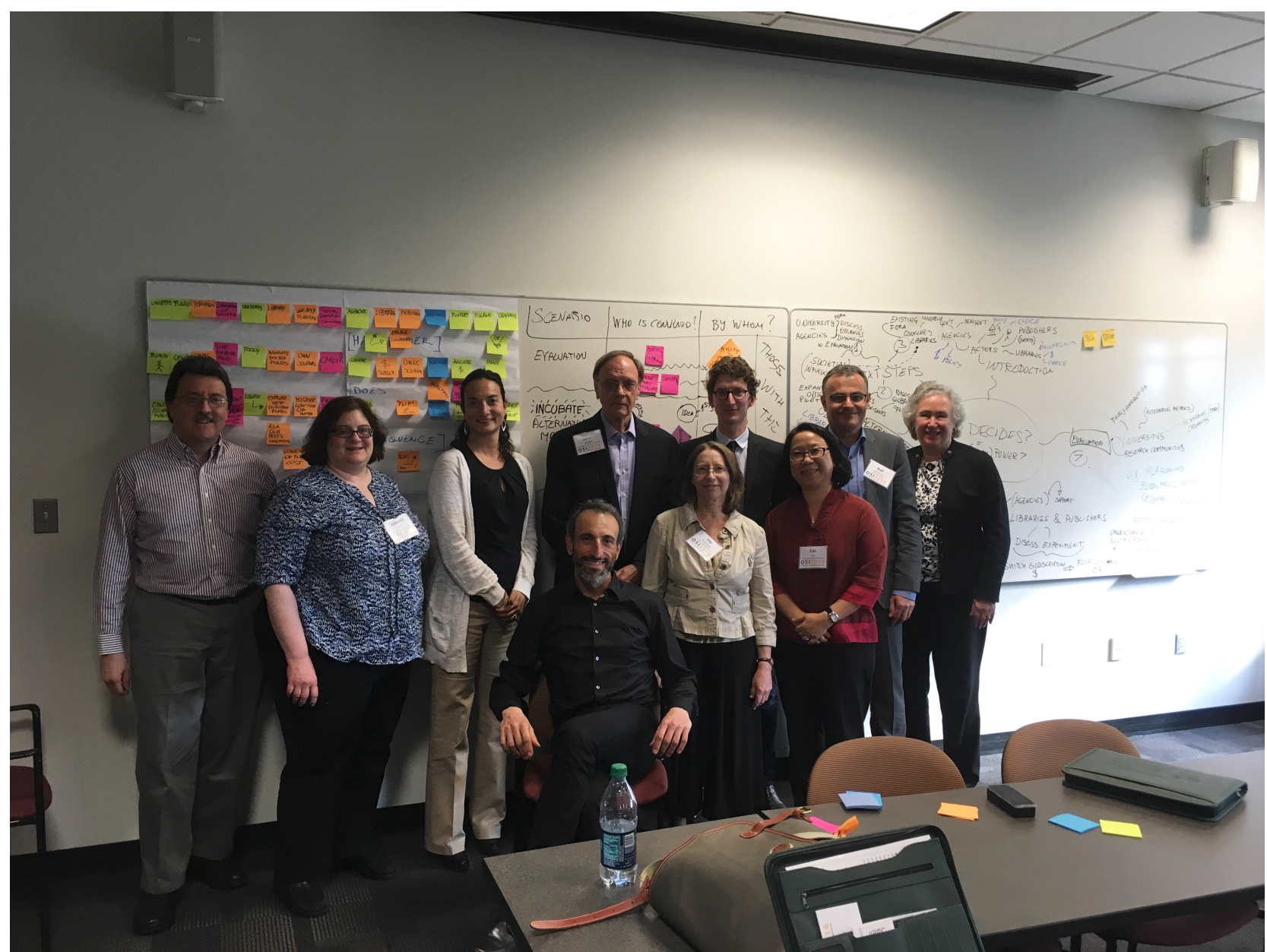

